\title{
Investigating insomnia as a cross-sectional and longitudinal predictor of loneliness: Findings from six samples
}

Melanie A. Hom ${ }^{\mathrm{a}, *}$, Jennifer L. Hames ${ }^{\mathrm{b}}$, Lindsay P. Bodell ${ }^{\mathrm{c}}$, Jennifer M. Buchman-Schmitt ${ }^{\mathrm{a}}$, Carol Chu $^{\mathrm{a}}$, Megan L. Rogers ${ }^{\mathrm{a}}$, Bruno Chiurliza ${ }^{\mathrm{a}}$, Matthew S. Michaels ${ }^{\mathrm{a}}$, Jessica D. Ribeiro ${ }^{\mathrm{a}}$, Michael R. Nadorff ${ }^{\mathrm{d}}$, E. Samuel Winer ${ }^{\mathrm{d}}$, Ingrid C. Lim ${ }^{\mathrm{e}}$, M. David Rudd ${ }^{\mathrm{f}}$, Thomas E. Joiner ${ }^{\mathrm{a}}$

a Department of Psychology, Florida State University, Tallahassee, FL, United States

b Department of Psychology, University of Notre Dame, Notre Dame, IN, United States

c Department of Psychiatry and Behavioral Neuroscience, University of Chicago, Chicago, IL, United States

a Department of Psychology, Mississippi State University, Starkville, MS, United States

e Office of the Army Surgeon General, Falls Church, VA, United States

${ }^{\mathrm{f}}$ Department of Psychology, University of Memphis, Memphis, TN, United States

\section{A R T I C L E I N F O}

\section{Keywords:}

Insomnia

Sleep disturbances

Sleep problems

Loneliness

Thwarted belongingness

\begin{abstract}
A B S T R A C T
Loneliness has been repeatedly associated with sleep problems; however, there is a dearth of research examining the prospective relationship between insomnia and loneliness, as well as this association controlling for other psychiatric symptoms. This study evaluated the cross-sectional and prospective relationship between insomnia and loneliness using six samples: 666 undergraduates; 2785 Army recruiters; 208 adults with a history of suicidality and/or depression; 343 adult psychiatric outpatients; 326 young adults at elevated suicide risk; and 183 undergraduates. A meta-analysis also was conducted to examine the magnitude of the relationship between insomnia and loneliness across the six studies. More severe insomnia symptoms were significantly associated with greater feelings of loneliness while accounting for some (e.g., anxiety, nightmares) but not all (i.e., depression) psychiatric covariates. Findings underscore the strength of the association between insomnia and loneliness and suggest that depression may account for this relationship. Additional studies are needed to further establish the temporal relationship between these variables, delineate the role of depression in the association between insomnia and loneliness, and test whether insomnia may confer unique risk for subsequent loneliness.
\end{abstract}

\section{Introduction}

Insomnia-characterized by the Diagnostic and Statistical Manual of Mental Disorders, $5^{\text {th }}$ Edition (DSM-5; American Psychiatric Association [APA], 2013) as "dissatisfaction with sleep quantity or quality, with complaints of difficulty initiating or maintaining sleep," and accompanying clinically significant distress or impairment (p. 363 )-is relatively common, with prevalence rates among the general population estimated at 6-15\% (Ford and Kamerow, 1989; Morin et al., 2006; Ohayon, 2002). At any given time, approximately one-third of individuals report experiencing at least one insomnia symptom (i.e., difficulty initiating or maintaining sleep, or early morning awakening; Morin et al., 2006).

Not only does insomnia contribute to daytime functional impairment (see Sateia et al. (2000), for review), but it also may be inherently distressing. For instance, the lengthy time spent in bed awake at night is often a treatment target since it may be particularly aversive and isolating (Edinger and Means, 2005). Indeed, it is plausible that the experience of insomnia itself may elicit feelings of loneliness (i.e., the painful perception and/or experience that one does not belong or is isolated from other individuals; Cacioppo and Patrick, 2008), which may account for findings suggesting that insomnia (see Bernert et al. (2015), and Pigeon et al. (2012), for review) and loneliness (c.f. thwarted belongingness; see Van Orden et al. (2010), for review) are each associated with similar negative psychiatric outcomes. Thus, empirical exploration of the relationship between insomnia and loneliness is indicated.

\subsection{The effect of insomnia on loneliness}

Few studies have investigated whether insomnia may increase risk for loneliness. In fact, even studies that have aimed to demonstrate a

\footnotetext{
* Corresponding author.

E-mail address: hom@psy.fsu.edu (M.A. Hom).
} 
prospective relationship between insomnia and loneliness have only tested this relationship through the use of proxy measures. For example, two studies by Roberts and colleagues $(2001 ; 2013)$ found that insomnia predicted difficulties in interpersonal functioning one year later among adolescents. Although poor interpersonal functioning may ultimately contribute to loneliness, they do not represent the same construct; thus, conclusions regarding whether insomnia influences loneliness remain speculative. To our knowledge, no additional research has examined this prospective relationship.

Despite the dearth of research in this domain, there are theoretical and empirical reasons why this prospective relationship may exist. First, as noted, being awake alone for many hours may be isolating and intrinsically lonely. Second, insomnia has been shown to impair daytime functioning (see Sateia et al. (2000), Shekleton et al. (2010), and Shochat et al. (2014), for review). These impairments may, in turn, impact individuals' motivation and/or ability to engage with others and build meaningful social connections. For example, evidence among adolescents supports that poor sleep is associated with difficulties forming and maintaining interpersonal relationships (Carskadon, 1990; Roberts et al., 2001; Roberts and Duong, 2013). Third, sleep is critical to emotion information processing (see Walker (2009), for review); thus, disturbed sleep may result in emotion regulation difficulties and greater negative affect. As a result, individuals experiencing insomnia may be more prone to maladaptive thoughts, including thoughts that they do not belong in social settings. Relatedly, individuals with insomnia demonstrate increased reactivity to daily stressors (Morin et al., 2003), which conceivably may increase reactivity to interpersonal conflicts or decrease their resources to attend to interpersonal needs. Concurrently, increased reactivity to daily stressors, alongside other insomnia-related consequences, might increase individuals' perceived need for social support.

Theoretical models of suicide and associated research also provide support for the notion that insomnia may lead to feelings of loneliness. The interpersonal theory of suicide (Joiner, 2005; Van Orden et al., 2010; cf. Joiner et al., 2016) posits that thwarted belongingness (i.e., lack of meaningful social connection) plays a critical role in the emergence of suicidal desire. Additionally, prior research has established a robust longitudinal relationship between insomnia and suicide risk (see Pigeon et al. (2012), for a meta-analytic review). Several crosssectional studies that have aimed to bridge the gap between this theory and research found that greater perceptions of thwarted belongingness significantly accounted for the relationship between insomnia symptoms and suicidal ideation (Chu et al., 2016, 2017; Hom et al., 2017). These findings suggest that loneliness may be one mechanism by which insomnia confers suicide risk. Consequently, further exploration of the prospective relationship between insomnia and loneliness is indicated, especially given the implications for other mental health domains.

\subsection{The effect of loneliness on sleep disturbances}

In contrast, a growing body of scientific literature supports the inverse-that loneliness may confer risk for the development of sleep problems. For example, studies have found that loneliness prospectively predicts poorer sleep efficiency (Cacioppo et al., 2002), higher levels of sleep fragmentation (Kurina et al., 2011), and poorer sleep quality (Harris et al., 2013). Notably, one study excluded individuals with mild to severe depression symptoms; suggesting that the relationship between loneliness and sleep problems is not significantly accounted for by depressive symptomatology (Cacioppo et al., 2002). Despite these robust findings, studies typically have not controlled for associated psychiatric symptoms (e.g., Cacioppo et al., 2002; Roberts et al., 2008), including depression and anxiety, which may explain these associations.

Several theories have been posited to explain how loneliness might contribute to sleep problems. It has been hypothesized that loneliness and a lack of a secure social environment lead to greater hypervigilance for social threats (Cacioppo and Cacioppo, 2014; Cacioppo and Hawkley, 2009). Consequently, the brain remains alert and prepared to detect and fight threats, even when an individual is trying to fall asleep or has just fallen asleep. Consistent with this hypothesis, animal models have indicated that social isolation may indeed disrupt neural mechanisms underlying sleep (see Cacioppo et al. (2014), for review). An alternate explanation posits that individuals who have more positive relationships or interactions with others are more likely to select healthy behaviors (Cohen and Wills, 1985). Because good sleep practices belong to the broader suite of healthy behaviors, individuals with greater social connection may be more likely to experience highquality sleep (Cacioppo et al., 2002).

\subsection{The present investigation}

Taken together, evidence suggests that loneliness is associated with and predicts subsequent sleep problems; however, to enhance our knowledge of the relationship between insomnia and loneliness, additional research is needed to test: (1) whether the association between insomnia and loneliness exists independent of other psychiatric symptoms (e.g., nightmares, anxiety, perceived burdensomeness), and (2) whether insomnia also predicts loneliness, controlling for other psychiatric symptoms. In this paper, we present findings from six studies that investigated the relationship between insomnia and loneliness both cross-sectionally (Studies 1-4) and prospectively (Studies 5 and 6). Additionally, we conducted a meta-analysis to examine the strength of the association between insomnia and loneliness across these six studies. Based on our findings, we employed a series of exploratory analyses for our prospective studies to further delineate the temporal relationships between insomnia, loneliness, and depression symptoms, as well as to better understand the directionality of effects.

Consistent with prior research and theory, we hypothesized that insomnia would be: (1) significantly associated with loneliness in crosssectional investigations, even after controlling for other psychiatric symptoms, and (2) a significant predictor of loneliness over time, controlling for other psychiatric symptoms. Similar to prior studies examining the relationship between loneliness and subsequent insomnia (e.g., Hawkley et al., 2010; Kurina et al., 2011), we elected to control for anxiety and/or depression symptoms where possible since they are likely to be associated with feelings of loneliness and also may confer risk for sleep disturbances.

Participants included undergraduates, military personnel, adults with a history of suicidality and/or depression, adult psychiatric outpatients, and young adults at elevated suicide risk. Each sample represents a group at elevated risk both for sleep disturbances (Bernert and Nadorff, 2015; Buysse et al., 2008; Owens et al., 2014; Seelig et al., 2010; Staner, 2010) and related psychiatric problems (Hoge et al., 2006; Kessler et al., 2014, 2005a; Ketchen Lipson et al., 2015; Mortier et al., 2017). Therefore, inclusion of each sample facilitated the examination of variables of interest in populations for whom research and clinical implications may be particularly salient. Multiple samples were also included in this investigation to enhance generalizability of findings and allow for meta-analytic evaluation. Of note, each study represents secondary analyses of data collected for other investigations. Thus, differences in measures of insomnia and loneliness, as well as available covariates, exist across studies. These differences allowed each study to either complement or build upon findings from other studies. The use of various self-report measures of insomnia and loneliness also bolstered our ability to identify whether these constructs have a conceptually meaningful relationship (i.e., as opposed to only finding a significant relationship between two specific measures). Institutional Review Board approval was obtained for each investigation, and all participants provided informed consent prior to participation. For ease of reference, demographic characteristics for each study and descriptive statistics for self-report measures are presented in Tables 1 and 2, respectively. 


\section{Study 1}

Study 1 tested the cross-sectional relationship between insomnia and loneliness, controlling for nightmares and anxiety, in a sample of undergraduate students.

\subsection{Method}

\subsubsection{Participants and procedures}

This sample included 747 undergraduate students from a large, public university in the Southern United States (U.S.). Of the total sample, 666 participants provided complete responses to all study measures and were included in analyses. There were no demographic differences between participants who did and did not complete all measures. Participants were recruited from an online survey sign-up portal. They completed self-report measures on a web-based portal and were compensated with course credit.

\subsubsection{Measures}

2.1.2.1. Insomnia Severity Index (ISI; Bastien et al., 2001). The ISI is a 7-item self-report measure that assesses the severity and consequences of individuals' insomnia symptoms over the past two weeks. Individuals rate the severity of their insomnia problems (e.g., "Difficulty falling asleep," "Difficulty staying asleep," "Problem waking up too early") and associated impairment and distress (e.g., "To what extent do you consider your sleep problem to interfere with your daily functioning?") on a 5 -point Likert scale. Total scores range from 0 to 28 , with increasing scores representing more severe symptoms. ${ }^{1}$ Prior studies indicate that the ISI has strong internal consistency and concurrent validity with other measures of sleep quality (Bastien et al., 2001; Morin et al., 2011). The ISI demonstrated good internal consistency in this study ( $\alpha=0.80)$.

2.1.2.2. Loneliness. Since a measure of loneliness was not included in the larger investigation, items from available measures (i.e., Center for Epidemiologic Studies Depression Scale (Radloff, 1977), PTSD CheckList (Weathers et al., 1991), Interpersonal Needs Questionnaire (Van Orden et al., 2012), and State Loss of Interest and Pleasure Scale (Winer et al., 2014b) were utilized to comprise an index of loneliness. Again, items were adjusted such that each item was equally weighted (i.e., scored on a 0-7 scale). Each item corresponds to an item on the well-validated UCLA Loneliness Scale (UCLA; Russell et al., 1980; see Supplementary Material for details). Total scores ranged from 0 to 49, with greater total scores representing greater perceptions of loneliness. In this sample, internal consistency was good $(\alpha=0.85)$.

2.1.2.3. Disturbing Dreams and Nightmare Severity Index (DDNSI; Krakow, 2006). The DDNSI is a 5-item measure of nightmare frequency, disruptiveness, severity, and intensity, each of which is factored into a total score that ranges from 0 to 37. A DDNSI total score greater than 10 indicates the potential presence of a nightmares disorder (Krakow et al., 2002). In the present sample, the DDNSI demonstrated good internal consistency $(\alpha=0.87)$.

2.1.2.4. Shortened Taylor Manifest Anxiety Scale (TMAS; Bendig, 1956). The shortened TMAS is a 20-item True/False measure of

\footnotetext{
${ }^{1}$ Due to an administration error, the first three items in Study 1 were scored on a $0-3$ scale $(0=$ No Problem; $3=$ Severe $)$ instead of a $0-4$ scale. These items were thus adjusted such that they were equally weighted as compared to items 4-7 (i.e., a score of 1 on item was multiplied by 1.33).
} 
Table 2

Means, standard deviations, normality statistics, and zero-order correlations for self-report measures.

\begin{tabular}{|c|c|c|c|c|c|c|c|c|c|c|c|c|}
\hline STUDY 1 & $\mathbf{1}$ & 2 & 3 & 4 & 5 & 6 & Range & M & $S D$ & Skew & Kurtosis & $a$ \\
\hline ISI Insomnia Symptoms (7-item) & - & & & & & & $0-24$ & 8.45 & 5.12 & 0.45 & -0.11 & 0.80 \\
\hline Loneliness & $0.38^{* *}$ & - & & & & & $5.40-42.75$ & 15.27 & 8.57 & 0.76 & -0.75 & 0.85 \\
\hline DDNSI Nightmares & $0.30^{* *}$ & $0.22^{* *}$ & - & & & & $0-26$ & 2.82 & 4.63 & 1.92 & 4.13 & 0.87 \\
\hline TMAS Manifest Anxiety & $0.45^{* *}$ & $0.57^{* *}$ & $0.27^{* *}$ & - & - & - & $0-20$ & 8.67 & 4.81 & 0.19 & -0.75 & 0.84 \\
\hline CES-D Depression Symptoms & $0.56^{* * *}$ & $0.67^{* *}$ & $0.32^{* * *}$ & $0.68^{* * *}$ & & & $0-51$ & 13.84 & 9.67 & 0.84 & 0.37 & 0.89 \\
\hline STUDY 2 & $\mathbf{1}$ & 2 & 3 & 4 & 5 & 6 & Range & $M$ & $S D$ & Skew & Kurtosis & $\alpha$ \\
\hline ISI Insomnia Symptoms (5-item) & - & & & & & & $0-20$ & 4.42 & 3.71 & 0.90 & 0.35 & 0.87 \\
\hline INQ Thwarted Belongingness (4-item) & $0.28^{* *}$ & - & & & & & $4-28$ & 7.14 & 4.44 & 2.11 & 5.42 & 0.90 \\
\hline INQ Perceived Burdensomeness (4-item) & $0.22^{* *}$ & $0.36^{* * *}$ & - & - & - & - & $4-28$ & 4.43 & 1.72 & 6.84 & 6.35 & 0.87 \\
\hline STUDY 3 & 1 & 2 & 3 & 4 & 5 & 6 & Range & $M$ & $S D$ & Skew & Kurtosis & $\alpha$ \\
\hline ISI Insomnia Symptoms (7-item) & - & & & & & & $7-28$ & 15.31 & 5.48 & 0.66 & -0.81 & 0.86 \\
\hline INQ Thwarted Belongingness (9-item) & $0.32^{* * *}$ & - & & & & & $9-59$ & 26.94 & 12.37 & 0.46 & -0.18 & 0.91 \\
\hline BAI Anxiety Symptoms & $0.50^{* *}$ & $0.34^{* *}$ & - & & & & $0-51$ & 12.27 & 8.66 & 1.26 & 2.02 & 0.87 \\
\hline BDI Depression Symptoms & $0.54^{* *}$ & $0.59^{* *}$ & $0.54^{* *}$ & - & - & - & $20-60$ & 29.20 & 7.65 & 1.46 & 2.81 & 0.88 \\
\hline STUDY 4 & 1 & 2 & 3 & 4 & 5 & 6 & Range & $M$ & $S D$ & Skew & Kurtosis & $\alpha$ \\
\hline ISI Insomnia Symptoms (7-item) & - & & & & & & $0-28$ & 10.88 & 6.60 & 0.26 & -0.58 & 0.88 \\
\hline INQ Thwarted Belongingness (9-item) & $0.27^{* *}$ & - & & & & & $9-63$ & 32.08 & 13.06 & -0.08 & -0.97 & 0.92 \\
\hline BAI Anxiety Symptoms & $0.46^{* *}$ & $0.31^{* * *}$ & - & & & & $0-59$ & 15.71 & 11.87 & 0.98 & 0.61 & 0.92 \\
\hline BDI Depression Symptoms & $0.51^{* *}$ & $0.61^{* * *}$ & $0.61^{* * *}$ & - & - & - & $0-56$ & 20.55 & 12.07 & 0.28 & -0.56 & 0.92 \\
\hline STUDY 6 & 1 & 2 & 3 & 4 & 5 & 6 & Range & $\mathbf{M}$ & $S D$ & Skew & Kurtosis & $\alpha$ \\
\hline HDSQ Insomnia Symptoms (T1) & - & & & & & & $0-8$ & 1.18 & 1.95 & 1.16 & 0.65 & 0.79 \\
\hline UCLA Loneliness (T1) & $0.44^{* *}$ & - & & & & & $0-66$ & 21.24 & 14.42 & 0.57 & -0.21 & 0.93 \\
\hline BDI Depression Symptoms (T1) & $0.52^{* * *}$ & $0.63^{* * *}$ & - & & & & $0-36$ & 7.00 & 7.60 & 1.58 & 2.51 & 0.91 \\
\hline STAI-State Anxiety Symptoms (T1) & $-0.34^{* *}$ & $-0.54^{* * *}$ & $-0.59^{* * *}$ & - & & & $12-60$ & 40.31 & 11.08 & -0.421 & -0.49 & 0.90 \\
\hline HDSQ Insomnia Symptoms (T2) & $0.61^{* * *}$ & $0.38^{* * *}$ & $0.41^{* *}$ & $-0.21^{*}$ & - & & $0-8$ & 1.52 & 1.93 & 1.91 & -0.46 & 0.87 \\
\hline UCLA Loneliness (T2) & $0.44^{* * *}$ & $0.78^{\text {*** }}$ & $0.57^{\text {*** }}$ & -0.47 & $0.49^{* * *}$ & - & $0-67$ & 20.20 & 15.80 & 0.65 & -0.47 & 0.95 \\
\hline
\end{tabular}

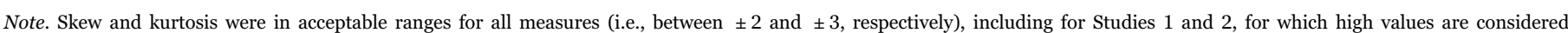

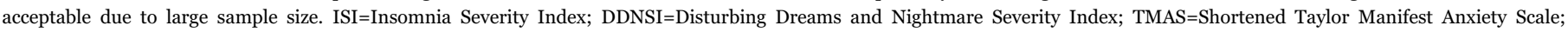

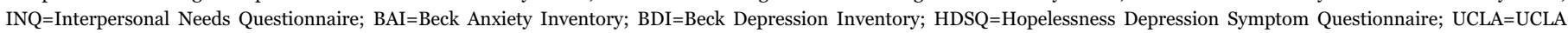
Loneliness Scale; T1=Baseline, T2=5-Week Follow-Up

${ }_{* * *}^{*} p<0.05$.

${ }^{* * *} p<0.01$.

anxiety symptoms (e.g., worry, feeling nervous). Total scores range from 0 to 20, with higher scores reflecting a greater tendency to experience anxiety. This abbreviated 20-item version of the TMAS was derived from the full 50-item measure (Taylor, 1953) and has been shown to have adequate reliability and greater clinical utility than the full-scale version (Bendig, 1956). In this sample, internal consistency was $\operatorname{good}(\alpha=0.84)$.

2.1.2.5. Center for Epidemiologic Studies Depression Scale (CES-D; Radloff, 1977). The CES-D is a 20-item measure of depression symptom severity. For this study, the CES-D item assessing loneliness was excluded from analyses; thus, possible total scores ranged from 0 to 57 and increasing scores indicated more severe symptoms. The CES-D has demonstrated strong psychometric properties across populations and settings (Radloff, 1977), and it and demonstrated strong internal consistency in this study $(\alpha=0.89)$.

\subsection{Results}

Hierarchical linear regression analysis revealed that greater ISI insomnia symptom severity was significantly associated with greater loneliness, even after controlling for DDNSI nightmares and TMAS manifest anxiety $\left(\beta=0.14, t[663]=3.75, p<0.01\right.$, partial $\left.r^{2}=0.02\right)$ but not after additionally controlling for CES-D depression $(\beta=-0.02$, $t[662]=-0.47, p=0.64$, partial $\left.r^{2}<0.01\right)$. This pattern remained controlling for sex, age, and race (see Supplementary Material).

\subsection{Discussion}

As expected, loneliness was associated with insomnia symptoms, beyond the effects of anxiety and nightmares. Though insomnia and nightmares share features (e.g., daytime functional impariment; Ohayon et al., 1997), findings suggest that unique components of insomnia may account for its relationship with loneliness. Furthermore, because many anxiety disorders are characterized both by overarousal and withdrawal (APA, 2013), it seems plausible that the relationship between insomnia and loneliness might be explained by anxiety symptoms. Thus, that insomnia and loneliness remained significantly associated even after controlling for anxiety symptoms underscores the strength of their relationship. However, effects did not remain significant after accounting for depression symptoms, suggesting that these symptoms may explain this relationship. Despite these promising findings, the cross-sectional study design precludes our ability to determine temporality of variables, the undergraduate sample limits the generalizability of findings, and other covariates were not available. Additionally, our measure of loneliness was not ideal (i.e., items pooled across measures) and its psychometric properties warrant further investigation in a sample in which a validated measure of loneliness can be utilized for comparison.

\section{Study 2}

To replicate these findings and examine additional covariates, in Study 2, we tested the cross-sectional relationship between insomnia and loneliness in a military sample, controlling for perceived burdensomeness, an associated construct.

\subsection{Method}

\subsubsection{Participants and procedures}

Participants were 2785 Army recruiters completing the Army Recruiting Course. Detailed procedures are described elsewhere (Ribeiro et al., 2015). 


\subsubsection{Measures}

3.1.2.1. ISI. An abbreviated 5-item version of the 7-item ISI was used to assess insomnia symptom severity. Total scores on this abbreviated measure ranged from 0 to 20 , with higher scores reflecting more severe insomnia symptoms. The abbreviated ISI demonstrated good internal consistency in the present sample ( $\alpha=0.87)$.

3.1.2.2. Interpersonal Needs Questionnaire (INQ; Van Orden et al., 2012). An abbreviated 8-item version of the 15 -item INQ was used in this study, with four items measuring thwarted belongingness (i.e., lack of meaningful social connection) and four perceived burdensomeness (i.e., belief that one is a burden on others). Both have been proposed as necessary and sufficient for suicidal desire (Joiner, 2005; Van Orden et al., 2010). Thwarted belongingness was utilized as an index of loneliness in this study. The INQ thwarted belongingness subscale has demonstrated significant and discriminant relations with the UCLA Loneliness Scale ( $r \mathrm{~s}=0.40-0.91$; Van Orden et al., 2012). Additionally, prior studies have similarly utilized the INQ thwarted belongingness subscale as an index of loneliness (Chu et al., 2016; Hom et al., 2017). Responses occur on a 7-point scale $(1=$ Not at all true for me; $7=$ Very true for me). The subscales demonstrated strong $(\alpha=0.90)$ and good internal consistency $(\alpha=0.87)$, respectively.

\subsection{Results}

Hierarchical linear regression analysis results are presented in Table 3. More severe ISI insomnia symptoms were associated with greater INQ thwarted belongingness, controlling for INQ perceived burdensomeness $\left(\beta=0.21, t[2722]=11.85, p<0.01\right.$, partial $\left.r^{2}=0.05\right)$. This pattern of findings remained after controlling for sex, age, and race (see Supplementary Material).

\subsection{Discussion}

Consistent with our hypothesis, insomnia symptom severity was associated with greater thwarted belongingness-an index of social disconnection and feelings of being an outsider (Van Orden et al., 2012). This association remained significant after controlling for INQ perceived burdensomeness, a construct highly correlated with depression symptoms (Cukrowicz et al., 2011; Jahn et al., 2011; Van Orden et al., 2008). This finding was notable given that insomnia is a key symptom of depression, and depression is often characterized by social withdrawal and perceptions of loneliness (APA, 2013). However, as with Study 1, Study 2's cross-sectional design limits our ability to draw conclusions regarding temporal relationships. Furthermore, our use of abbreviated self-report measures (ISI, INQ) and the INQ perceived burdensomeness subscale as a proxy for depressions symptoms may have impacted our findings. Finally, our sample represented a specialized military subgroup, limiting generalizability.

\section{Study 3}

Since depression and suicidality have both been shown to be associated with insomnia (see Baglioni et al. (2011), and Bernert et al. (2015), for review), we aimed to replicate our findings in an adult student and community sample with a history of suicidality and depression.

\subsection{Method}

4.1.1. Participants and procedures

A total of 208 adults with a history of suicidality and/or a major
Table 3

Hierarchical linear regression analyses examining the association between insomnia and loneliness.

\begin{tabular}{|c|c|c|c|c|c|}
\hline & $R^{2}$ & $\beta$ & $t$ & $p$ & partial $r^{2}$ \\
\hline \multicolumn{6}{|l|}{ STUDY 1 (DV = Loneliness) } \\
\hline Step 1: & 0.14 & & & $<0.01$ & \\
\hline ISI Insomnia Symptoms & & 0.38 & 10.44 & $<0.01$ & 0.14 \\
\hline Step 2: & 0.15 & & & $<0.01$ & \\
\hline ISI Insomnia Symptoms & & 0.34 & 9.05 & $<0.01$ & 0.11 \\
\hline DDNSI Nightmares & & 0.12 & 3.06 & $<0.01$ & 0.01 \\
\hline Step 3: & 0.34 & & & $<0.01$ & \\
\hline ISI Insomnia Symptoms & & 0.14 & 3.75 & $<0.01$ & 0.02 \\
\hline DDNSI Nightmares & & 0.05 & 1.34 & 0.18 & $<0.01$ \\
\hline TMAS Manifest Anxiety & & 0.50 & 13.85 & $<0.01$ & 0.23 \\
\hline Step 4: & 0.45 & & & $<0.01$ & \\
\hline ISI Insomnia Symptoms & & -0.02 & -0.47 & 0.64 & $<0.01$ \\
\hline DDNSI Nightmares & & 0.01 & 0.22 & 0.82 & $<0.01$ \\
\hline TMAS Manifest Anxiety & & 0.22 & 5.50 & $<0.01$ & 0.04 \\
\hline CES-D Depression Symptoms & & 0.51 & 11.77 & $<0.01$ & 0.18 \\
\hline \multicolumn{6}{|c|}{ STUDY 2 (DV = INQ Thwarted Belongingness) } \\
\hline Step 1: & 0.08 & & & $<0.01$ & \\
\hline ISI Insomnia Symptoms & & 0.28 & 15.28 & $<0.01$ & 0.08 \\
\hline Step 2: & 0.17 & & & $<0.01$ & \\
\hline ISI Insomnia Symptoms & & 0.21 & 11.85 & $<0.01$ & 0.05 \\
\hline INQ Perceived Burdensomeness & & 0.31 & 17.57 & $<0.01$ & 0.10 \\
\hline \multicolumn{6}{|c|}{ STUDY 3 (DV = INQ Thwarted Belongingness) } \\
\hline Step 1: & 0.10 & & & $<0.01$ & \\
\hline ISI Insomnia Symptoms & & 0.32 & 4.77 & $<0.01$ & 0.10 \\
\hline Step 2: & 0.14 & & & $<0.01$ & \\
\hline ISI Insomnia Symptoms & & 0.20 & 2.69 & 0.01 & 0.04 \\
\hline BAI Anxiety Symptoms & & 0.23 & 3.08 & $<0.01$ & 0.05 \\
\hline Step 3: & 0.35 & & & & \\
\hline ISI Insomnia Symptoms & & $<-0.01$ & -0.05 & 0.96 & $<0.01$ \\
\hline BAI Anxiety Symptoms & & 0.02 & 0.32 & 0.75 & $<0.01$ \\
\hline BDI Depression Symptoms & & 0.58 & 8.01 & $<0.01$ & 0.25 \\
\hline \multicolumn{6}{|c|}{ STUDY 4 (DV = INQ Thwarted Belongingness) } \\
\hline Step 1: & 0.07 & & & $<0.01$ & \\
\hline ISI Insomnia Symptoms & & 0.27 & 5.20 & $<0.01$ & 0.07 \\
\hline Step 2: & 0.12 & & & $<0.01$ & \\
\hline ISI Insomnia Symptoms & & 0.16 & 2.82 & 0.01 & 0.02 \\
\hline BAI Anxiety Symptoms & & 0.24 & 4.10 & $<0.01$ & 0.05 \\
\hline Step 3: & 0.38 & & & $<0.01$ & \\
\hline ISI Insomnia Symptoms & & -0.03 & -0.63 & 0.53 & $<0.01$ \\
\hline BAI Anxiety Symptoms & & -0.09 & -1.61 & 0.11 & 0.01 \\
\hline BDI Depression Symptoms & & 0.68 & 12.03 & $<0.01$ & 0.30 \\
\hline
\end{tabular}

Note. Variance inflation factors (VIFs) were in acceptable ranges for all analyses (i.e., all VIFs < 3). DV=Dependent Variable; ISI=Insomnia Severity Index; DDNSI=Disturbing Dreams and Nightmare Severity Index; TMAS=Shortened Taylor Manifest Anxiety Scale; INQ=Interpersonal Needs Questionnaire; BAI=Beck Anxiety Inventory; BDI=Beck Depression Inventory.

depressive episode were included in Study 3. Participants were recruited from a large university in the Southeastern U.S. and the surrounding community. Individuals completed a web-based screening survey and were invited to participate if they reported a lifetime history of: (a) $\geq 1$ suicide attempt, (b) suicidal ideation that included a plan, and/or (c) a major depressive episode. Of note, $26.1 \%$ reported a suicide attempt history, 36.4\% a history of suicide plans, 90.3\% a history of suicidal ideation, $80.7 \%$ a history of a major depressive episode, and $46.4 \%$ a history of non-suicidal self-injury. Eligible participants completed self-report surveys during a laboratory study visit. All participants were provided with a list of mental health resources.

\subsubsection{Measures}

4.1.2.1. ISI. In this sample, the 7-item ISI demonstrated good internal consistency $(\alpha=0.86)$. 
4.1.2.2. INQ-TB. The 9-item thwarted belongingness subscale (INQ$\mathrm{TB}$ ) of the 15-item INQ was utilized as a loneliness index. INQ-TB scores range from 7 to 63 ; higher scores indicate greater thwarted belongingness. In this study, the INQ-TB's internal consistency was excellent $(\alpha=0.91)$.

4.1.2.3. Beck Anxiety Inventory (BAI; Beck et al., 1988). The BAI is a 21-item self-report inventory designed to assess level of subjective anxiety within the past week. Responses are rated on a 3-point Likert scale $(0=$ Not at all; $3=$ Severely, $I$ could barely stand it), and total scores range from 0 to 63 , with higher scores indicating more severe anxiety symptoms. In this sample, reliability of the BAI was good $(\alpha=0.87)$.

4.1.2.4. Beck Depression Inventory (BDI-II; Beck and Steer, 1987). The BDI-II is a 21-item self-report measure of depression symptom severity, with higher scores indicating greater severity. The BDI-II has been found to have strong psychometric properties across samples (Beck and Steer, 1987). In this study, the BDI-II was used without the sleep item and demonstrated good internal consistency $(\alpha=0.88)$.

\subsection{Results}

Hierarchical linear regression analyses results are presented in Table 3. ISI insomnia symptom severity was significantly associated with INQ thwarted belongingness, even when controlling for BAI anxiety symptoms $\left(\beta=0.20, t[200]=2.69 ; p=0.01\right.$, partial $\left.r^{2}=0.04\right)$; however, not after additionally controlling for BDI-II depression symptoms $\left(\beta<-0.01, t[199]=-0.05 ; p=0.96\right.$, partial $\left.r^{2}<0.01\right)$. To determine whether the inclusion of two covariates in a single model accounted for this BDI-II finding, an additional model was examined in which only BDI-II depression symptoms were controlled for; however, this association still was not significant in this model $(\beta<0.01, t[200]$ $=0.05 ; p=0.96$, partial $\left.r^{2}<0.01\right)$. This pattern of findings remained the same after including sex, race, and age as covariates (see Supplementary Material).

\subsection{Discussion}

Building upon and consistent with results of Studies 1 and 2, findings from this study provide evidence that more severe insomnia symptoms are associated with greater loneliness, even among individuals with a history of suicidality and/or depression. One primary aim of understanding the relationship between insomnia and loneliness is to inform the development of prevention and intervention strategies that may thwart the trajectory from sleep disturbances to other psychopathology (e.g., mood disorders, suicide risk). Importantly, these findings establish a relationship between insomnia and loneliness in a sample at elevated risk for psychiatric problems and, consequently, for whom such research is relevant and critical. As with Study 1, this relationship remained significant even when accounting for the effects of anxiety but not depression symptoms, suggesting that depression symptoms, specifically, may explain this association. Similar to Studies 1 and 2, this study's cross-sectional design limited our ability to infer the direction of the relationship. Additionally, though this study included individuals with a history of psychiatric problems, work is needed to test these relationships in a clinical sample.

\section{Study 4}

To address the limitations of Study 3, we sought to replicate previous findings in a clinical sample of adult outpatients receiving care at a community-based mental health clinic.

\subsection{Method}

\subsubsection{Participants and procedures}

A total of 343 adult outpatients at a university psychology training clinic in the Southeastern U.S. participated in Study 4. Individuals presenting to this clinic are only referred elsewhere if they are at imminent suicide or homicide risk, or have psychotic and/or manic symptoms not stabilized by psychiatric medications. Thus, with the clinic's sliding scale fees, its outpatients have a broad range of clinical presentations, including severe psychopathology and suicide risk. Prior to initiating services, all participants completed self-report questionnaires.

\subsubsection{Measures}

5.1.2.1. ISI. The ISI demonstrated good internal consistency in this sample $(\alpha=0.88)$.

5.1.2.2. INQ-TB. The INQ-TB demonstrated strong internal consistency in this study ( $\alpha=0.92)$.

5.1.2.3. BAI. The BAI was shown to have excellent internal consistency ( $\alpha=0.92)$.

5.1.2.4. BDI-II (Beck and Steer, 1987). The BDI-II was used without the sleep item and demonstrated good internal consistency $(\alpha=0.92)$.

\subsection{Results}

See Table 3 for results from hierarchical linear regression analyses. More severe ISI insomnia symptoms were associated with significantly higher levels of INQ-TB thwarted belongingness $(\beta=0.27, t[341]=5.20$, $p<0.01$, partial $r^{2}=0.07$ ), including after controlling for BAI anxiety symptoms $\left(\beta=0.16, t[340]=2.82, p<0.01\right.$, partial $\left.r^{2}=0.02\right)$ but not after additionally controlling for BDI depression symptoms $(\beta=-0.03$, $t[339]=-0.63, p=0.53$, partial $\left.r^{2}<0.01\right)$. Again, to examine whether the inclusion of two covariates in a single model accounted for this finding, BDI depression symptoms were included as the sole covariate in the model, but the association still was not significant $(\beta=-0.05$, $t[340]=-1.03, p=0.31$, partial $r^{2}<0.01$ ). Similar to prior studies, this pattern of results remained the same after controlling for sex, race, and age (see Supplementary Material).

\subsection{Discussion}

The association found between ISI insomnia symptoms and INQ thwarted belongingness, including when controlling for anxiety symptoms, is consistent with findings from Studies 1 to 3. Similar to Study 3, effects did not remain significant after accounting for depression symptoms, further indicating that these symptoms may explain the association between insomnia and thwarted belongingness. However, that insomnia and loneliness were correlated among mental health outpatients suggests that monitoring and assessing both constructs during treatment may be clinically useful. Given prior work highlighting a prospective relationship between loneliness and sleep disturbances, the bolstering of social support and interpersonal connections (e.g., behavioral activation; Martell et al., 2001) may also therapeutically impact insomnia symptoms. Additional work is war- 
ranted to replicate these findings among other adult outpatient samples. Furthermore, as with Studies 1-3, the current study only examined the cross-sectional relationship between insomnia and loneliness, rather than the prospective relationship.

\section{Study 5}

Given the limitations of our prior cross-sectional study designs, in Study 5, we examined sleep problems as a predictor of loneliness in a sample of young adults at elevated suicide risk.

\subsection{Method}

\subsubsection{Participants and procedures}

This sample consisted of 326 young adults who presented to a military medical center in acute suicidal crisis and elected to enroll in an outpatient suicide prevention treatment trial for suicidal young adults (Rudd et al., 1996). Following informed consent, participants completed self-report questionnaires, which were completed again 1, 6, 12,18 , and 24 months later. Longitudinal analyses only examined the 1-month $(n=235)$ and 6-months $(n=143)$ follow-up periods given the high rates of study attrition (73\%) beginning at 12-months follow-up.

\subsubsection{Measures}

6.1.2.1. Sleep problems. A single item from the Beck Depression Inventory (BDI; Beck et al., 1961) was used to assess sleep problems on a 4-point scale $(0=$ "I have not experienced any change in my sleeping pattern"; 1= "I sleep somewhat more than usual" or "I sleep somewhat less than usual"; $2=$ "I sleep a lot more than usual" or "I sleep a lot less than usual"; and 3="I sleep most of the day" or "I wake up 1-2 hours early and can't get back to sleep").

6.1.2.2. Suicide Probability Scale (SPS; Cull and Gill, 1989). Loneliness was assessed using the SPS, a 36-item self-report measure designed to measure attitudes and behaviors associated with suicide risk. The SPS has demonstrated good psychometric properties, including strong test-retest reliability (Cull and Gill, 1995). For this study, nine items from the SPS were used to form a loneliness subscale (see Supplementary Material). Items are rated on a 4-point scale ( $0=$ None of the time; $3=$ All of the time $)$ and weighted from 0 to 5 . Total weighted scores for this subscale range from 0 to 45 , with higher scores representing a greater degree of loneliness. This loneliness subscale demonstrated adequate to good internal consistency across time points ( $\alpha=0.69-0.83$ ). The SPS has been previously utilized as a proxy for thwarted belongingness (Joiner et al., 2009).

6.1.2.3. BDI-I (Beck et al., 1961). The BDI-I demonstrated strong internal consistency across study time points $(\alpha=0.91-0.95)$.

\subsection{Results}

Means, standard deviations, and zero-order correlations for selfreport measures are presented in Table 4 and 5. Cross-sectional analyses revealed that sleep problems and loneliness severity were significantly associated at each of the six study time points $(r \mathrm{~s}=0.62-$ 0.74 , ps $<0.05$; see Table 5). Hierarchical linear regression analyses revealed that greater BDI sleep problems significantly predicted higher levels of SPS loneliness both at 1 -month $(\beta=0.17, t[234]=2.59, p=0.01$, partial $\left.r^{2}=0.03\right)$ and 6-months $(\beta=0.18, t[142]=2.22, p=0.03$, partial $r^{2}=0.03$ ) follow-up. However, this significant finding was not maintained when controlling for baseline SPS loneliness severity $(\beta=0.02$,
Table 4

Study 5 means and standard deviations of measures at each time point.

\begin{tabular}{lcccccc}
\hline & $\boldsymbol{M}$ & SD & Range & Skew & Kurtosis & $\boldsymbol{\alpha}$ \\
\hline SPS - Loneliness Subscale & & & & & & \\
Baseline & 18.29 & 5.81 & $7-32$ & 0.08 & 0.27 & 0.76 \\
1 Month & 15.74 & 5.22 & $7-31$ & 0.42 & 0.31 & 0.70 \\
6 Months & 15.05 & 5.69 & $7-32$ & 0.82 & 0.40 & 0.78 \\
12 Months & 14.89 & 5.74 & $7-29$ & 0.69 & 0.52 & 0.78 \\
18 Months & 15.11 & 6.17 & $7-31$ & 0.93 & 0.70 & 0.83 \\
24 Months & 13.32 & 4.34 & $8-25$ & 1.53 & 0.90 & 0.69 \\
BDI - Sleep Item & & & & & & - \\
Baseline & 1.22 & 0.96 & $0-3$ & -0.70 & 0.27 & - \\
1 Month & 0.63 & 0.63 & $0-3$ & 0.93 & 0.31 & - \\
6 Months & 0.47 & 0.47 & $0-3$ & 1.42 & 0.39 & - \\
12 Months & 0.57 & 0.57 & $0-3$ & 1.58 & 0.51 & - \\
18 Months & 0.50 & 0.50 & $0-3$ & 1.36 & 0.67 & - \\
24 Months & 0.50 & 0.50 & $0-2$ & -0.13 & 0.83 & - \\
BDI - Total (No Sleep Item) & & & & & & - \\
Baseline & 18.39 & 11.36 & $0-43$ & 0.21 & -0.92 & 0.91 \\
1 Month & 9.44 & 9.31 & $0-38$ & 1.10 & 0.41 & 0.91 \\
6 Months & 8.09 & 8.72 & $0-50$ & 1.52 & 3.15 & 0.91 \\
12 Months & 7.16 & 9.35 & $0-44$ & 1.99 & 4.08 & 0.93 \\
18 Months & 8.53 & 11.02 & $0-44$ & 1.97 & 3.59 & 0.95 \\
24 Months & 5.32 & 8.01 & $0-31$ & 2.11 & 4.26 & 0.93 \\
\hline
\end{tabular}

Note. Skew and kurtosis were in acceptable ranges for all self-report measures across time points utilized in regression analyses; SPS=Suicide Probability Scale; BDI=Beck Depression Inventory.

Table 5

Study 5 cross-sectional associations between insomnia and loneliness at each time point.

\begin{tabular}{ll}
\hline & SPS Loneliness \\
\hline BDI Sleep Problems (Baseline) & $0.62^{* *}$ \\
BDI Sleep Problems (1 Month) & $0.62^{* * *}$ \\
BDI Sleep Problems (6 Months) & $0.74^{* *}$ \\
BDI Sleep Problems (12 Months) & $0.70^{* * *}$ \\
BDI Sleep Problems (18 Months) & $0.69^{* * *}$ \\
BDI Sleep Problems (24 Months) & $0.60^{* *}$ \\
\hline
\end{tabular}

Note. BDI=Beck Depression Inventory

$$
{ }^{* * *} p<0.01 \text {. }
$$

$t[233]=0.31, p=0.75$, partial $r^{2}<0.01 ; \beta=0.06, t[141]=0.79, p=0.43$, partial $\left.r^{2}<0.01\right)$ and both baseline SPS loneliness and BDI depression symptoms $\left(\beta=0.01, t[232]=0.13, p=0.89\right.$, partial $r^{2}<0.01 ; \beta=0.08$, $t[140]=0.99, p=0.33$, partial $\left.r^{2}=0.01\right)$ at $1-$ and 6 -months follow-up, respectively (see Table 6). Baseline BDI depression symptoms did not significantly predict SPS loneliness in either of these models (see Table 6).

To evaluate the directionality of these relationships, regression analyses were utilized to examine whether baseline SPS loneliness symptoms might predict BDI sleep problems. Greater SPS loneliness symptoms did not predict BDI sleep problem severity either at 1-month $\left(\beta=0.11, t[239]=1.69, p=0.09\right.$, partial $\left.r^{2}=0.01\right)$ or 6 -months $(\beta=0.09$, $t[147]=1.12, \quad p=0.27$, partial $\left.r^{2}=0.01\right)$ follow-up, including when controlling for baseline BDI sleep problems only $(\beta=0.02, t[238]$ $=0.27, p=0.79$, partial $r^{2}<0.01 ; \beta=0.03, t[146]=0.34, p=0.74$, partial $\left.r^{2}<0.01\right)$ and both baseline BDI sleep problems and BDI depression symptoms $\quad\left(\beta=-0.02, \quad t[237]=-0.22, \quad p=0.83, \quad\right.$ partial $r^{2}<0.01$; $\beta=-0.02, t[145]=-0.18, p=0.86$, partial $r^{2}<0.01$ ) at $1-$ and 6 -months follow-up. Baseline BDI depression symptoms did not significantly predict SPS loneliness in either of these models. The same pattern of results emerged when controlling for study treatment condition and sex, race, and age (see Supplementary Material).

Follow-up analyses were conducted first in an effort to understand whether depression symptoms might serve as a causal link between insomnia and loneliness. Bootstrap mediation analyses revealed that 1month BDI depression symptoms did not significantly mediate the relationship between baseline BDI insomnia symptoms and 6-month 
Table 6

Hierarchical linear regression analyses examining the prospective relationship between insomnia and loneliness.

\begin{tabular}{|c|c|c|c|c|c|c|c|}
\hline & $R^{2}$ & $\Delta R^{2}$ & $\Delta F$ & $\beta$ & $t$ & $p$ & partial $r^{2}$ \\
\hline \multicolumn{8}{|c|}{ STUDY 5 (1-Month Follow-Up; DV = SPS Loneliness) } \\
\hline Step 1: & 0.03 & 0.03 & $3.34^{*}$ & & & 0.01 & \\
\hline BDI Sleep Problems (T1) & & & & 0.17 & 2.59 & 0.01 & 0.03 \\
\hline Step 2: & 0.25 & 0.22 & $66.71^{* * *}$ & & & $<0.01$ & \\
\hline BDI Sleep Problems (T1) & & & & 0.02 & 0.31 & 0.75 & $<0.01$ \\
\hline SPS Loneliness (T1) & & & & 0.49 & 8.17 & $<0.01$ & 0.22 \\
\hline Step 3: & 0.25 & $<0.01$ & 0.15 & & & $<0.01$ & \\
\hline BDI Sleep Problems (T1) & & & & 0.01 & 0.13 & 0.89 & $<0.01$ \\
\hline SPS Loneliness (T1) & & & & 0.47 & 6.48 & $<0.01$ & 0.15 \\
\hline BDI Depression Symptoms (T1) & & & & 0.03 & 0.35 & 0.73 & $<0.01$ \\
\hline \multicolumn{8}{|c|}{ STUDY 5 (6-Month Follow-Up; DV = SPS Loneliness) } \\
\hline Step 1: & 0.03 & 0.03 & 2.59 & & & 0.03 & \\
\hline BDI Sleep Problems (T1) & & & & 0.18 & 2.22 & 0.03 & 0.03 \\
\hline Step 2: & 0.23 & 0.19 & $34.88^{* * *}$ & & & $<0.01$ & \\
\hline BDI Sleep Problems (T1) & & & & 0.06 & 0.79 & 0.43 & $<0.01$ \\
\hline SPS Loneliness (T1) & & & & 0.46 & 5.96 & $<0.01$ & 0.20 \\
\hline Step 3: & 0.23 & $<0.01$ & 0.41 & & & $<0.01$ & \\
\hline BDI Sleep Problems (T1) & & & & 0.08 & 0.99 & 0.33 & 0.01 \\
\hline SPS Loneliness (T1) & & & & 0.50 & 4.96 & $<0.01$ & 0.15 \\
\hline BDI Depression Symptoms (T1) & & & & -0.07 & -0.65 & 0.52 & $<0.01$ \\
\hline \multicolumn{8}{|c|}{ STUDY 6 (5-Weeks Follow-Up; DV = UCLA Loneliness) } \\
\hline Step 1: & 0.19 & 0.19 & $35.34^{* *}$ & & & $<0.01$ & \\
\hline HDSQ Insomnia Symptoms (T1) & & & & 0.44 & 5.95 & $<0.01$ & 0.19 \\
\hline Step 2: & 0.61 & 0.42 & $160.87^{* *}$ & & & $<0.01$ & \\
\hline HDSQ Insomnia Symptoms (T1) & & & & 0.12 & 2.15 & 0.03 & 0.03 \\
\hline UCLA Loneliness (T1) & & & & 0.73 & 12.68 & $<0.01$ & 0.52 \\
\hline Step 3: & 0.62 & $<0.01$ & 0.75 & & & $<0.01$ & \\
\hline HDSQ Insomnia Symptoms (T1) & & & & 0.12 & 2.01 & 0.05 & 0.03 \\
\hline UCLA Loneliness (T1) & & & & 0.70 & 10.80 & $<0.01$ & 0.44 \\
\hline STAI-State Anxiety Symptoms (T1) & & & & -0.05 & -0.87 & 0.39 & 0.01 \\
\hline Step 4: & 0.62 & $<0.01$ & 1.34 & & & $<0.01$ & \\
\hline HDSQ Insomnia Symptoms (T1) & & & & 0.09 & 1.55 & 0.12 & 0.02 \\
\hline UCLA Loneliness (T1) & & & & 0.67 & 9.59 & $<0.01$ & 0.39 \\
\hline STAI-State Anxiety Symptoms (T1) & & & & -0.03 & -0.41 & 0.68 & $<0.01$ \\
\hline BDI Depression Symptoms (T1) & & & & 0.08 & 1.10 & 0.27 & 0.01 \\
\hline
\end{tabular}

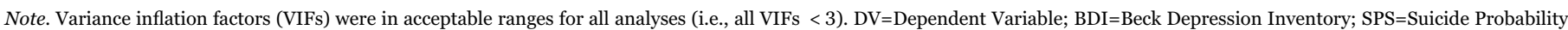
Scale; HDSQ=Hopelessness Depression Symptom Questionnaire; UCLA=UCLA Loneliness Scale; T1=Baseline

${ }^{*} p<0.05$.

${ }^{* * *} p<0.01$

SPS loneliness, controlling for baseline BDI depression symptoms and SPS loneliness (95\% CI: $-0.75,1.17)$. Furthermore, to delineate further the temporal relationships between study constructs, additional hierarchical linear regression analyses were conducted. Analyses revealed that neither baseline BDI insomnia symptoms $(p=0.22)$ nor baseline SPS loneliness $(p=0.95)$ significantly predicted 1-month BDI depression symptoms, controlling for baseline BDI depression symptoms. Although baseline BDI insomnia symptoms did not significantly predict more severe 6-month BDI depression symptoms, controlling for baseline BDI depression symptoms $(p=0.93)$, greater baseline SPS loneliness did $\left(\beta=-0.34, t[145]=3.10, p<0.01\right.$, partial $\left.r^{2}=0.05\right)$.

\subsection{Discussion}

Consistent with Studies 1 through 4, sleep problems and loneliness were significantly correlated at each time point. Sleep problems also significantly predicted higher levels of loneliness 1- and 6- months later. However, this prospective relationship only remained significant when baseline loneliness was not included as a covariate; thus, preexisting loneliness may be an especially robust predictor of subsequent loneliness. The bidirectional nature of the association between loneliness and sleep problems may have contributed to difficulties establishing a temporal relationship between these symptom clusters. Interestingly, though, depression symptoms did not mediate the relationship between insomnia and subsequent loneliness, suggesting that these symptoms did not account for this observed relationship.
Despite prior research demonstrating that loneliness is a strong predictor of future insomnia symptoms and other sleep problems (Cacioppo et al., 2002; Harris et al., 2013), this result was not replicated here. This may be the result of the nature of our sample and use of an item that both measures insomnia and hypersomnia. Similar to insomnia, hypersomnia may negatively impact social functioning (Jennum et al., 2014); however, due to the nature of data collected, we were not able to separately examine the effects of insomnia and hypersomnia on loneliness, challenging interpretability of our findings. Finally, our ability to identify sleep problems as a significant predictor of loneliness may have been challenged by the use of proxy measures of study constructs. Further studies are thus indicated to address these limitations.

\section{Study 6}

In Study 6, we examined the extent to which insomnia symptoms would predict feelings of loneliness levels five weeks later among a sample of undergraduates. We also tested the reverse relationship (i.e., loneliness predicting subsequent insomnia symptoms).

\subsection{Method}

\subsubsection{Participants and procedures}

A total of 183 undergraduate students enrolled in a general psychology course at a large, public university in the Southeastern 
U.S. participated in Study 6. Participants completed questionnaires at baseline and five weeks later. Only 151 participants (82.5\%) completed all items at both time points.

\subsubsection{Measures}

7.1.2.1. Sleep Hopelessness Depression Symptom Questionnaire (HDSQ; Metalsky and Joiner, 1997). The HDSQ is a 32-item selfreport measure designed to assess symptoms associated with hopelessness depression. Individuals rate each item on a 4-point scale. The HDSQ has demonstrated high internal consistency and good predictive validity (Metalsky and Joiner, 1997). For this study, four items assessing insomnia symptoms (i.e., difficulties falling asleep, sustaining sleep, and problems with early waking) were used to create an insomnia subscale. Total scores ranged from 0 to 12, with higher scores indicating more severe symptoms. The four items of the HDSQ used in this study demonstrated adequate to good internal consistency $(\alpha=0.79-0.87)$.

7.1.2.2. UCLA Loneliness Scale (UCLA; Russell et al., 1980). The UCLA is a 20-item self-report measure that assesses perceptions of loneliness. In the current study, a 5-point Likert scale ranging from 0 (never) to 4 (often) was utilized. Total scores ranged from 0 to 80 with higher scores indicative of increased feelings of loneliness. ${ }^{2}$ Past studies have shown that the UCLA has adequate test-retest reliability and good concurrent validity (Russell et al., 1980). In the current study, the UCLA demonstrated strong internal consistency ( $\alpha=0.93-$ 0.95).

7.1.2.3. State Trait Anxiety Inventory - State Subscale (STAI-State; Spielberger, 1983). The STAI-State is a 20 item self-report measure that assesses current anxiety symptoms. Participants were asked to rank each item on a 4-point Likert scale ranging from 0 (almost never) to 3 (almost always). Total scores range from 0 to 60 with higher scores signifying higher levels of state anxiety. The STAI has been found to have high internal consistency, adequate test-retest reliability, and good construct and concurrent validity (Spielberger, 1989, 1983). The alpha coefficient of the STAI in the current study was 0.90 , indicating good reliability.

7.1.2.4. BDI-II. The BDI was used without the sleep item and demonstrated good internal consistency $(\alpha=0.91)$.

\subsection{Results}

Missing data were addressed using listwise deletion, and the final sample included only participants who completed all items at both time points $(n=151)$. No significant demographic or symptom differences existed between those excluded from and included in analyses. Scaled scores were calculated for baseline UCLA, STAI-State, and BDI-II, and for the HDSQ insomnia subscale at 5-weeks follow-up. Outliers (zscore $>|3.3|$ ) were brought to the upper acceptable limit to reduce undue influence on the observed relationship between insomnia and loneliness (Warner, 2013). Hierarchical linear regression analysis revealed that baseline HDSQ insomnia symptoms significantly predicted UCLA loneliness five weeks later, controlling for baseline UCLA loneliness and STAI-State anxiety symptoms $(\beta=0.12, t[148]=2.01$,

\footnotetext{
${ }^{2}$ Traditionally, the UCLA utilizes a four-point Likert scale (1-4). However, for the larger investigation, researchers elected to impose a five-point Likert scale in order to maintain consistency with other measures.
}

$p=0.05$, partial $r^{2}=0.03$ ), but not when also controlling for BDI-II depression symptoms $\left(\beta=0.09, t[147]=1.55, p=0.12\right.$, partial $\left.r^{2}=0.02\right)$. Though, BDI-II depression symptoms did not significantly predict UCLA loneliness in this model (see Table 6). When only controlling for baseline UCLA loneliness and BDI-II depression symptoms, the prospective relationship between HDSQ insomnia symptoms and UCLA loneliness was not significant $(\beta=0.09, t[148]=1.57, p=0.12$, partial $r^{2}=0.02$ ).

Similarly, for the reverse relationship designed to test the directionality of these associations, analyses indicated that baseline UCLA loneliness significantly predicted greater HDSQ insomnia symptoms five weeks later, controlling for baseline HDSQ insomnia symptoms and STAI-State anxiety $\left(\beta=0.18, t[148]=2.17, p=0.03\right.$, partial $\left.r^{2}=0.03\right)$, but not when also controlling for BDI-II depression symptoms $(\beta=0.13$, $t[147]=1.45 p=0.14$, partial $\left.r^{2}=0.05\right)$. Again, though, BDI-II depression symptoms did not significantly predict HDSQ insomnia symptoms in this model $(p=0.22)$. When only controlling for baseline HDSQ insomnia symptoms and BDI-II depression symptoms, the relationship between UCLA loneliness and subsequent HDSQ insomnia was not significant $\left(\beta=0.11, t[148]=1.28, p=0.20\right.$, partial $\left.r^{2}=0.01\right)$. Due to limitations in access to data, we were unable to control for sex, race, and age in our analyses.

Follow-up analyses were conducted to evaluate additional prospective relationships between variables to better understand their temporal associations. Hierarchical linear regression analyses revealed that neither baseline HDSQ insomnia symptoms $(p=0.41)$ nor baseline UCLA loneliness $(p=0.23)$ significantly predicted BDI-II depression symptoms at follow-up, controlling for baseline BDI-II depression symptoms.

\subsection{Discussion}

Overall, Study 6's findings suggest that a prospective relationship between insomnia and loneliness exists, even after accounting for baseline loneliness and anxiety symptoms but not after controlling for depression symptoms. These findings are consistent with Study 5's results. Though insomnia symptoms may be a robust predictor of loneliness, it is not surprising that depression-of which a key feature is anhedonia (i.e., lack of interest in activities previously enjoyed; APA 2013; Winer et al., 2014a)-may account for this relationship.

Consistent with prior work supporting the prospective relationship between loneliness and subsequent insomnia (Cacioppo et al., 2002; Hawkley et al., 2010), loneliness significantly predicted insomnia symptoms across a five-week period, controlling for baseline insomnia and anxiety symptoms but not depression symptoms. Based on these findings, it is hypothesized that the relationship between insomnia and loneliness is cyclical. For example, insomnia may lead to increased loneliness, and subsequently, loneliness may exacerbate insomnia. Research is needed to determine the interplay of these symptoms over time. Overall, these findings largely support our hypotheses and are consistent with aforementioned work (Mauss et al., 2013; Morin et al., 2003; Roberts and Duong, 2013; Shochat et al., 2014). Although Study 6's prospective design represents a study strength, our young adult sample limits the generalizability of our findings.

\section{Meta-analysis of studies}

To further consolidate the effects found across these six studies, we conducted a meta-analysis of bivariate correlations between insomnia symptoms and loneliness from each sample.

\subsection{Method}

Pearson's $r$ bivariate correlations were recorded for each study using unadjusted estimates. Effect sizes were transformed to Fisher's zs for the purpose of analysis, but the effect sizes presented were re- 
transformed to Pearson's $r$ for ease of interpretation. A random effects model was utilized to allow for heterogeneity across studies (e.g., variations in study design and measurement); a random effects model also allows for a greater degree of generalizability to other populations. Effect sizes were weighted, and we examined the significance of the Qtest to determine whether there was substantial heterogeneity of effect sizes. We also report the $I^{2}$ statistic, which indicates how much of the variability across studies is due to heterogeneity as opposed to chance due to sampling error $(0.25=$ small heterogeneity; $0.50=$ medium heterogeneity; $0.75=$ large heterogeneity). Given the nature of the studies, we expected this value to be relatively high. We also examined whether results were significantly moderated by the samples' mean age, percentage of females, percentage identifying as White/Caucasian, and mean depression symptom scores. Regarding dependent effect sizes, Studies 5 and 6 reported multiple effects across time points. To include the most conservative effects in our meta-analysis, we used only one effect per sample. Specifically, in Study 5, the longitudinal effect of insomnia at baseline and loneliness at 6-month follow-up was utilized; in Study 6, we included the longitudinal effect of insomnia at time 1 and loneliness at time 2.

\subsection{Results}

Effect sizes and 95\% CIs are presented in a forest plot (see Fig. 1). The Q-test was significant (14.27), and the $I^{2}$ value (0.65) indicated that a moderate-to-large degree of variability was due to heterogeneity rather than chance. The test of the null was significant, with a moderate effect size $(r=0.32, p<0.01,95 \%$ CI $[0.22,0.42])$. This indicates that across studies, the bivariate association between insomnia and loneliness is significant and moderate in magnitude. These results were moderated by age $(Z=-2.12, p=0.03)$, sex $(Z=9.31, p<0.01)$, race $(Z=5.47, p<0.01)$, and depression symptom severity $(Z=-5.91, p<$ $0.01)$. Effects were stronger for younger participants, samples with a higher percentage of females, samples with higher percentages of participants identifying as White/Caucasian, and samples with lower depression symptom levels.

\subsection{Discussion}

These results further underscore the strength of the relationship between insomnia and loneliness. With regard to the age moderation finding, it is possible that younger individuals may be more likely to be experiencing the first onset of sleep disturbances (Kessler et al., 2005b; National Sleep Foundation Sleep, 2000); thus, the experience of insomnia may be especially distressing and isolating. Little is known about differences in experiences of insomnia between sexes and

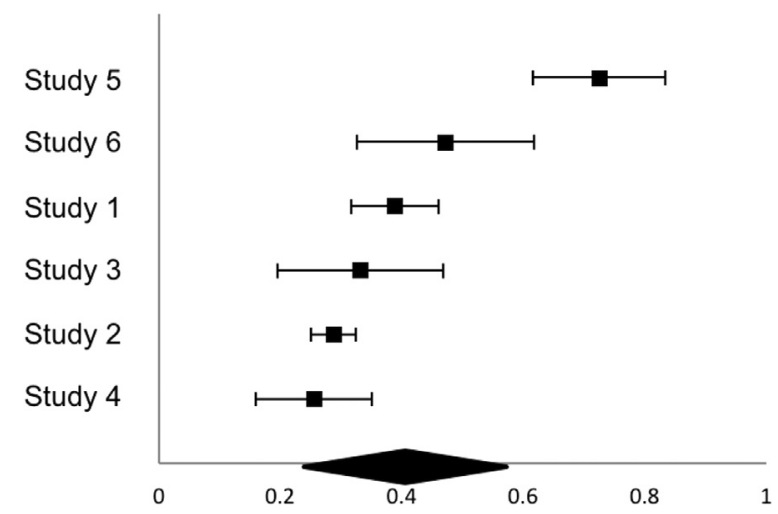

Fig. 1. Forest plot of Fisher's z effect sizes from meta-analysis of the association between insomnia and loneliness. Note: Study $1=$ Undergraduate sample; Study 2=Army recruiter sample; Study $3=$ Adults with a prior history of suicidality and/or depression; Study 4=Adult outpatient sample; Study $5=$ Young adults at elevated suicide risk; Study 6=Undergraduate sample. cultural groups; thus, the finding that the association between insomnia and loneliness was stronger in samples with greater proportions of females and those identifying as White/Caucasian warrants further investigation. It is possible, for instance, that perceptions of social support or the effects of inadequate sleep may differ between groups. Finally, the finding that insomnia symptoms were more strongly associated with loneliness in samples with lower mean depression symptom scores is consistent with a prior study demonstrating a robust association between these constructs in a sample that excluded individuals with mild to severe depression symptoms (Cacioppo et al., 2002). However, further research is indicated to understand what factors may underlie these findings. It may be, for example, that the varying time frames utilized to assess study constructs influenced results (e.g., insomnia and depression symptoms were often assessed over the past two weeks, but loneliness was assessed "recently" or as a stable state). Given the limited number of samples included in moderation analyses ( $N=6$ for demographic variable analyses; $N=4$ for depression symptoms analyses), we recommend that these findings be interpreted with caution and replicated across other samples. Relatedly, moderation analyses examining the role of study design and measurement tools in yielding observed effects may be useful in future investigations that include additional samples. These analyses may be especially informative in studies that utilize standardized validated loneliness scales, such as the UCLA (e.g., only utilized in Study 6).

\section{General discussion}

In this investigation, we examined both cross-sectional and prospective associations between insomnia and increased feelings of loneliness in six distinct samples. Meta-analytic results and other findings across studies not only provide converging preliminary evidence that more severe insomnia symptoms are associated with greater feelings of loneliness, but also add to the growing body of research on psychological consequences of sleep problems.

Consistent with study hypotheses and prior research, insomnia and loneliness were significantly associated across all six study samples. Given the marked diversity across samples with respect to sociodemographic characteristics and clinical severity, it is noteworthy that this significant association emerged for each study, even when controlling for sociodemographic variables. Though the mechanisms underlying this relationship cannot be ascertained from this investigation, the aforementioned theoretical and empirical explanations for this association remain plausible, including that insomnia may be intrinsically lonely. Further work is needed, though, to directly test if these proposed mechanisms hold explanatory power.

Contrary to hypotheses, the association between insomnia and loneliness did not remain significant after controlling for depression symptoms in Study 1 and Studies 3 through 6 . These findings emphasize the primacy of depression and suggest that depression symptomatology may account for the relationship between insomnia and loneliness. This may be due, in part, to the highly overlapping nature of insomnia and depression (i.e., insomnia is a depression symptom), as exemplified in a finding that approximately $85 \%$ of outpatients who meet criteria for major depressive disorder reported clinically significant sleep disturbances (Zimmerman et al., 2015). Inclusion of depression as a covariate also may have resulted in overadjustment, and similar to suicidal ideation with depression covaried out (Rogers et al., 2016), the conceptual significance of insomnia with depression covaried out may be limited. Loneliness is also likely both associated with and a consequence of depression. Even so, it is worthwhile to note that loneliness itself is not a symptom of depression and, consequently, our findings do not solely embody a clustering of depression symptoms. Loneliness also has been significantly associated with insomnia even among individuals without depression (Cacioppo et al., 2002). Therefore, though depression may have largely explained 
the association between insomnia and loneliness in our investigation, this pattern of findings may have resulted from our use of samples of relatively high clinical severity (i.e., mean depression measure scores in the mild to severe range).

Research also indicates that insomnia often precedes depression (Baglioni et al., 2011); thus, with the addition of assessment points and use of at-risk, subclinical samples, the temporal relationships between insomnia, loneliness, and depression may be further delineated. For instance, insomnia and loneliness may together precede and predict depression onset. Indeed, in Study 5, loneliness was identified as a significant predictor of depression at 6-months follow-up, even after controlling for baseline depression symptoms. A lack of significant findings for other follow-up analyses, however, suggests that further work is needed. Additionally, our study only examined effects up to 6 months later, and it is possible that given a longer time frame, as previous studies have utilized (e.g., Baglioni and Riemann, 2012; Cacioppo et al., 2002), a different pattern of results may have emerged. Depression may also serve as a common cause of loneliness or causal intermediate in the relationship between insomnia and loneliness, possibly explaining its effects on the relationship between insomnia and loneliness. Though depression did not emerge as a significant mediator of this relationship in Study 5, research is needed to replicate these findings utilizing improved measures of insomnia and loneliness. Rigorous assessment of specific depression and insomnia symptoms may be especially illuminating.

Importantly, the association between insomnia and loneliness remained significant even when controlling for nightmares, anxiety symptoms, and/or perceived burdensomeness, each of which is strongly associated with insomnia and/or loneliness. Findings suggest that the association between insomnia and loneliness is not being driven solely by these other psychiatric symptoms and does not simply represent a clustering of anxious features or perceptions of burdensomeness. These results are noteworthy and underscore the specific directionality and robustness of the association between insomnia and loneliness. As a result, the conjecture that unique features of insomnia (i.e., hours awake in social isolation) may exacerbate or confer risk for loneliness particularly warrants further empirical investigation, especially in clinical populations where these relationships may have greater relevance.

The significant prospective relationship found between insomnia and loneliness across two samples represents a novel finding. These results support a growing body of literature highlighting the negative consequences of poor sleep (see Sateia et al. (2000), for review). The lack of significant results for the reverse relationship for Study 5 (i.e., loneliness as a predictor of insomnia) was particularly surprising, especially given scientific evidence that such a relationship exists (Cacioppo et al., 2002; Harris et al., 2013; Hawkley et al., 2010). Differences in study design, participant samples, and assessment techniques may, in part, account for these equivocal findings. It is also possible that insomnia and loneliness may be associated via distinct pathways that depend on the direction of the relationship and population being investigated.

On this point, the prospective relationship between insomnia and loneliness found in Studies 5 and 6 signals a need for studies examining whether loneliness may serve a mediating role in the relationship between insomnia and other psychiatric problems. For example, by increasing risk for thwarted belongingness, a key component of suicidal desire (Joiner, 2005; Van Orden et al., 2010), insomnia may increase risk for suicide. This is consistent with findings from aforementioned studies identifying thwarted belongingness as a mediator of the association between insomnia and suicidal symptoms (Chu et al., 2017, 2016; Hom et al., 2017).

Lastly, findings from this investigation suggest that it may be useful to examine whether therapeutically impacting loneliness and/or depression may thwart the trajectory from insomnia to other negative psychiatric outcomes. Fortunately, efficacious interventions exist that may support the enhancement of social support and interpersonal connections, as well as target depression symptoms (e.g., behavioral activation; Martell et al., 2001). Studies also suggest that concurrent insomnia-specific treatment for individuals receiving depression treatment may not only improve sleep but also enhance depression outcomes (Clarke et al., 2015; Manber et al., 2011, 2008). Insomnia treatments (e.g., CBT for insomnia; Edinger and Means, 2005) can be delivered in group settings and thus may have the added benefit of providing an outlet to build social relationships and, as a result, reduce loneliness.

\subsection{Limitations and future directions}

Our investigation approach was limited in several ways. First, we were only able to examine the prospective relationship between insomnia and loneliness in two of six samples. Both of these samples were composed of young adults; thus, findings should be replicated in other sociodemographic groups, particularly in older adults who often display high levels of insomnia, loneliness, and suicidality (Bernert et al., 2014; Cukrowicz et al., 2011; Jaremka et al., 2014). In so doing, temporal and causal relationships between insomnia and loneliness can be more rigorously examined. Insomnia symptoms and loneliness also remained relatively stable over time in Studies 5 and 6 (i.e., no statistically significant differences between mean scores at baseline and follow-up for participants included in prospective analyses; $p s>0.05$ ), limiting our interpretation of findings. Second, some self-report measures may not have fully captured the constructs they were intended to measure. For example, in some studies, abbreviated versions were used (Study 2), items were combined across measures (Study 1) and within a single measure (Studies 5 and 6) to assess a specific construct, and proxy measures were utilized to assess constructs of interest (Studies 3 and 4). In some cases, these measurement approaches may have impacted our ability to detect significant results and challenged our ability to compare our findings to previous studies utilizing standardized measures. Consequently, future studies would benefit from more direct and thorough assessments of constructs of interest, including additional demographic covariates (e.g., marital status; Luhmann and Hawkley, 2016). Similarly, because these studies were secondary analyses of larger investigations, we were unable to include the same constructs across studies, limiting our ability to draw conclusions across samples. Fourth, all studies relied on self-report measures, which are subject to biases. Future studies may benefit from the inclusion of objective measures of sleep quality and quantity (e.g., polysomnography, actigraphy). Finally, our investigation did not allow for the direct test of why insomnia might confer risk for the development of loneliness. Although we offer several possible explanations, the studies in this investigation were, for example, unable to probe whether individuals suffering from insomnia report the specific experience of being awake when others are asleep as distressing. As a result, additional research is needed to identify the processes that may explain the relationship between insomnia and subsequent loneliness, with specific efforts to determine whether these awake-hours are experienced as being intrinsically socially isolating.

\subsection{Conclusions}

In summary, findings suggest that more severe insomnia symptoms are associated with and may prospectively predict greater feelings of loneliness, even after controlling for relevant covariates such as nightmares, anxiety symptoms, and perceptions of burdensomeness. However, this association-including when evaluated prospectivelydid not remain significant after accounting for depression symptoms. As such, further work is needed to identify and delineate the unique features of insomnia and depression that increase risk for subsequent loneliness, particularly in clinical populations for whom findings will be most salient. We look forward to additional studies that address the 
limitations of this current investigation, which represents a promising initial step toward understanding the relationship between these constructs.

\section{Declaration of interest}

Conflicts of interest: none.

\section{Acknowledgments}

This research was supported in part by grants from the Military Suicide Research Consortium, an effort supported by the Office of the Assistant Secretary of Defense for Health Affairs under Award No. (W81XWH-10-2-0181), the National Institute of Mental Health (MH48097; T32MH18921; 5T32MH09331104; T32MH18921; T32MH082761), and the John Templeton Foundation via the Greater Good Science Center at University of California, Berkeley (Subagreement 00008137). Opinions, interpretations, conclusions, and recommendations are those of the author and are not necessarily endorsed by the Department of Defense, Department of Veterans Affairs, MSRC, National Institute of Mental Health, or John Templeton Foundation. The funding sources did not have any role in the study design; in the collection, analysis and interpretation of data; in the writing of the report; or in the decision to submit the article for publication.

\section{References}

American Psychiatric Association, 2013. Diagnostic and Statistical Manual of Mental DisordersDSM-5 5th edition. American Psychiatric Association, Washington, DC.

Baglioni, C., Battagliese, G., Feige, B., Spiegelhalder, K., Nissen, C., Voderholzer, U., Lombardo, C., Riemann, D., 2011. Insomnia as a predictor of depression: a metaanalytic evaluation of longitudinal epidemiological studies. J. Affect. Disord. 135, 10-19. http://dx.doi.org/10.1016/j.jad.2011.01.011.

Baglioni, C., Riemann, D., 2012. Is chronic insomnia a precursor to major depression? Epidemiological and biological findings. Curr. Psychiatry Rep. 14, 511-518. http:// dx.doi.org/10.1007/s11920-012-0308-5.

Bastien, C.H., Vallieres, A., Morin, C.M., Vallières, A., Morin, C.M., 2001. Validation of the Insomnia Severity Index as an outcome measure for insomnia research. Sleep Med. 2, 297-307. http://dx.doi.org/10.1016/S1389-9457(00)00065-4.

Beck, A.T., Epstein, N., Brown, G., Steer, R.A., 1988. An inventory for measuring clinical anxiety: psychometric properties. J. Consult. Clin. Psychol. 56, 893-897. http:// dx.doi.org/10.1037//0022-006x.56.6.893.

Beck, A.T., Steer, R.A., 1987. Manual for the Revised Beck Depression Inventory. Psychological Corporation, San Antonio, TX.

Beck, A.T., Ward, C.H., Mendelson, M., Mock, J., Erbaugh, J., 1961. An inventory for measuring depression. Arch. Gen. Psychiatry 4, 561-571. http://dx.doi.org/ 10.1001/archpsyc.1961.01710120031004.

Bendig, A.W., 1956. The development of a short form of the manifest anxiety scale. J. Consult. Psychol. 20, 384.

Bernert, R.A., Kim, J.S., Iwata, N.G., Perlis, M.L., 2015. Sleep disturbances as an evidence-based suicide risk factor. Curr. Psychiatry Rep. 17, 1-9. http://dx.doi.org/ 10.1007/s11920-015-0554-4.

Bernert, R.A., Nadorff, M.R., 2015. Sleep disturbances and suicide risk. Sleep Med. Clin. 10, 35-39. http://dx.doi.org/10.1016/j.jsmc.2014.11.004

Bernert, R.A., Turvey, C.L., Conwell, Y., Joiner, T.E., 2014. Association of poor subjective sleep quality with risk for death by suicide during a 10-year period: a longitudinal, population-based study of late life. JAMA Psychiatry 71, 1129-1137. http:// dx.doi.org/10.1001/jamapsychiatry.2014.1126.

Buysse, D.J., Angst, J., Gamma, A., Ajdacic, V., Eich, D., Rössler, W., 2008. Prevalence, course, and comorbidity of insomnia and depression in young adults. Sleep 31, $473-480$.

Cacioppo, J.T., Cacioppo, S., 2014. Social relationships and health: the toxic effects of perceived social isolation. Soc. Personal. Psychol. Compass 8, 58-72. http:// dx.doi.org/10.1111/spc3.12087.

Cacioppo, J.T., Hawkley, L.C., 2009. Perceived social isolation and cognition. Trends Cogn. Sci. 13, 447-454. http://dx.doi.org/10.1016/j.tics.2009.06.005.

Cacioppo, J.T., Hawkley, L.C., Berntson, G.G., Ernst, J.M., Gibbs, A.C., Stickgold, R., Hobson, J.A., 2002. Do lonely days invade the nights? Potential social modulation of sleep efficiency. Psychol. Sci. 13, 384-387. http://dx.doi.org/10.1111/j.0956- 7976.2002.00469.x.

Cacioppo, J.T., Patrick, W., 2008. Loneliness: Human Nature and the Need for Social Connection. WWNorton \& Co., New York, NY.

Cacioppo, S., Capitanio, J.P., Cacioppo, J.T., 2014. Toward a neurology of loneliness. Psychol. Bull. 140, 1464-1504. http://dx.doi.org/10.1037/a0037618.

Carskadon, M.A., 1990. Patterns of sleep and sleepiness in adolescents. Pediatrician 17 $5-12$.

Chu, C., Hom, M.A., Rogers, M.L., Ringer, F.B., Hames, J.L., Suh, S., Joiner, T.E., 2016. Is insomnia lonely? Exploring thwarted belongingness as an explanatory link between insomnia and suicidal ideation in a sample of South Korean university students. J. Clin. Sleep Med..

Chu, C., Hom, M.A., Rogers, M.L., Stanley, I.H., Ringer-Moberg, F.B., Podlogar, M.C., Hirsch, J.K., Joiner, T.E., 2017. Insomnia and suicide-related behaviors: a multistudy investigation of thwarted belongingness as a distinct explanatory factor. J. Affect. Disord. 208, 153-162. http://dx.doi.org/10.1016/j.jad.2016.08.065.

Clarke, G., McGlinchey, E.L., Hein, K., Gullion, C.M., Dickerson, J.F., Leo, M.C., Harvey, A.G., 2015. Cognitive-behavioral treatment of insomnia and depression in adolescents: a pilot randomized trial. Behav. Res. Ther. 69, 111-118. http:// dx.doi.org/10.1016/j.brat.2015.04.009.

Cohen, S., Wills, T.A., 1985. Stress, social support, and the buffering hypothesis. Psychol. Bull. 98, 310-357. http://dx.doi.org/10.1037//0033-2909.98.2.310.

Cukrowicz, K.C., Cheavens, J.S., Van Orden, K.A., Ragain, R.M., Cook, R.L., 2011. Perceived burdensomeness and suicide ideation in older adults. Psychol. Aging 26, 331-338. http://dx.doi.org/10.1037/a0021836.

Cull, J., Gill, W., 1989. The Suicide Probability Scale Manual. Western Psychological Services, Los Angeles, CA.

Cull, J.G., Gill, W.S., 1995. The suicide probability scale manual.

Edinger, J.D., Means, M.K., 2005. Cognitive-behavioral therapy for primary insomnia. Clin. Psychol. Rev. 25, 539-558. http://dx.doi.org/10.1016/j.cpr.2005.04.003.

Ford, D.E., Kamerow, D.B., 1989. Epidemiologic study of sleep disturbances and psychiatric disorders. An opportunity for prevention? J. Am. Med. Assoc. 262, (1479-1184).

Harris, R.A., Qualter, P., Robinson, S.J., 2013. Loneliness trajectories from middle childhood to pre-adolescence: impact on perceived health and sleep disturbance. J. Adolesc. 36, 1295-1304. http://dx.doi.org/10.1016/j.adolescence.2012.12.009.

Hawkley, L.C., Preacher, K.J., Cacioppo, J.T., 2010. Loneliness impairs daytime functioning but not sleep duration. Heal. Psychol. 29, 124-129. http://dx.doi.org/ $10.1037 / \mathrm{a} 0018646$.

Hoge, C.W., Auchterlonie, J.L., Milliken, C.S., 2006. Mental health problems, use of mental health services, and attrition from military service after returning from deployment to Iraq or Afghanistan. J. Am. Med. Assoc. 295, 1023-1032. http:// dx.doi.org/10.1001/jama.295.9.1023.

Hom, M.A., Chu, C., Schneider, M.E., Lim, I.C., Hirsch, J.K., Gutierrez, P.M., Joiner, T.E., 2017. Thwarted belongingness as an explanatory link between insomnia symptoms and suicidal ideation: findings from three samples of military service members and veterans. J. Affect. Disord. 209, 114-123. http://dx.doi.org/10.1016/ j.jad.2016.11.032.

Jahn, D.R., Cukrowicz, K.C., Linton, K., Prabhu, F., 2011. The mediating effect of perceived burdensomeness on the relation between depressive symptoms and suicide ideation in a community sample of older adults. Aging Ment. Health 15, 214-220. http://dx.doi.org/10.1080/13607863.2010.501064.

Jaremka, L.M., Andridge, R.R., Fagundes, C.P., Alfano, C.M., Povoski, S.P., Lipari, A.M., Agnese, D.M., Arnold, M.W., Farrar, W.B., Yee, L.D., Carson, W.E., Bekaii-Saab, T., Martin, E.W., Schmidt, C.R., Kiecolt-Glaser, J.K., 2014. Pain, depression, and fatigue: loneliness as a longitudinal risk factor. Heal. Psychol. 33, 948-957. http:// dx.doi.org/10.1037/a0034012.

Jennum, P., Ibsen, R., Avlund, K., Kjellberg, J., 2014. Health, social and economic consequences of hypersomnia: a controlled national study from a national registry evaluating the societal effect on patients and their partners. Eur. J. Heal. Econ. 15, 303-311. http://dx.doi.org/10.1007/s10198-013-0491-2.

Joiner, T.E., 2005. Why People Die by Suicide. Harvard University Press, Cambridge, MA. http://dx.doi.org/10.5860/choice.44-1002.

Joiner, T.E., Hom, M.A., Hagan, C.R., Silva, C., 2016. Suicide as a derangement of the self-sacrificial aspect of eusociality. Psychol. Rev.. http://dx.doi.org/10.1037/ rev0000020.

Joiner, T.E., Van Orden, K.A.A., Witte, T.K.K., Selby, E.A.A., Ribeiro, J.D.D., Lewis, R., Rudd, M.D.D., Joiner, T.E., Jr., Van Orden, K.A.A., Witte, T.K.K., Selby, E.A.A., Ribeiro, J.D.D., Lewis, R., Rudd, M.D.D., 2009. Main predictions of the interpersonal-psychological theory of suicidal behavior: empirical tests in two samples of young adults. J. Abnorm. Psychol. 118, 634-646. http://dx.doi.org/ $10.1037 / \mathrm{a} 0016500$.

Kessler, R.C., Berglund, P., Demler, O., Jin, R., Merikangas, K.R., Walters, E.E., 2005a. Lifetime prevalence and age-of-onset distributions of DSM-IV disorders in the National Comorbidity Survey Replication. Arch. Gen. Psychiatry 62, 593-602. http://dx.doi.org/10.1001/archpsyc.62.6.593.

Kessler, R.C., Berglund, P., Demler, O., Jin, R., Merikangas, K.R., Walters, E.E., 2005b. Lifetime prevalence and age-of-onset distributions of DSM-IV disorders in the National Comorbidity Survey Replication. Arch. Gen. Psychiatry 62, 593-602. http://dx.doi.org/10.1001/archpsyc.62.6.593.

Kessler, R.C., Heeringa, S.G., Stein, M.B., Colpe, L.J., Fullerton, C.S., Hwang, I., Naifeh, J.A., Nock, M.K., Petukhova, M., Sampson, N.A., Schoenbaum, M., Zaslavsky, A.M., Ursano, R.J., 2014. Thirty-day prevalence of DSM-IV mental disorders among nondeployed soldiers in the US Army: Results from the Army Study to Assess Risk and Resilience in Servicemembers (Army STARRS). JAMA Psychiatry 71, 504-513. http://dx.doi.org/10.1001/jamapsychiatry.2014.28.

Ketchen Lipson, S., Gaddis, S.M., Heinze, J., Beck, K., Eisenberg, D., 2015. Variations in 
student mental health and treatment utilization across US colleges and universities. J. Am. Coll. Heal. 63, 388-396. http://dx.doi.org/10.1080/ 07448481.2015 .1040411$.

Krakow, B., 2006. Nightmare complaints in treatment-seeking patients in clinical sleep medicine settings: diagnostic and treatment implications. Sleep 29, 1313-1319.

Krakow, B., Schrader, R., Tandberg, D., Hollifield, M., Koss, M.P., Yau, C.L., Cheng, D.T., 2002. Nightmare frequency in sexual assault survivors with PTSD. J. Anxiety Disord. 16, 175-190. http://dx.doi.org/10.1016/s0887-6185(02)00093-2.

Kurina, L.M., Knutson, K.L., Hawkley, L.C., Cacioppo, J.T., Lauderdale, D.S., Ober, C., 2011. Loneliness is associated with sleep fragmentation in a communal society. Sleep 34, 1519-1526. http://dx.doi.org/10.5665/sleep.1390.

Luhmann, M., Hawkley, L.C., 2016. Age differences in loneliness from late adolescence to oldest old age. Dev. Psychol. 52, 943-959. http://dx.doi.org/10.1037/dev0000117.

Manber, R., Bernert, R.A., Suh, S., Nowakowski, S., Siebern, A.T., Ong, J.C., 2011. CBT for insomnia in patients with high and low depressive symptom severity: adherence and clinical outcomes. J. Clin. Sleep Med. 7, 645-652. http://dx.doi.org/10.5664/ jesm. 1472.

Manber, R., Edinger, J., Gress, J., San Pedro-Salcedo, M., Kuo, T., Kalista, T., 2008. Cognitive behavioral therapy for insomnia enhances depression outcome in patients with comorbid major depressive disorder and insomnia. Sleep 31, 489-495.

Martell, C.R., Addis, M.E., Jacobsen, N.S., 2001. Depression in Context: Strategies for Guided Action. WWNorton \& Co.

Mauss, I.B., Troy, A.S., LeBourgeois, M.K., 2013. Poorer sleep quality is associated with lower emotion-regulation ability in a laboratory paradigm. Cogn. Emot. 27, 567-576 . http://dx.doi.org/10.1080/02699931.2012.727783.

Metalsky, G.I., Joiner, T.E.J., 1997. The hopelessness depression symptom questionnaire. Cogn. Ther. Res. 21, 359-384. http://dx.doi.org/10.1023/ A: 1021882717784 .

Morin, C.M., Belleville, G., Bélanger, L., Ivers, H., Belanger, L., Ivers, H., 2011. The insomnia severity index: psychometric indicators to detect insomnia cases and evaluate treatment response. Sleep 34, 601-608. http://dx.doi.org/10.1111/j.13652648.2010.05394.x.

Morin, C.M., LeBlanc, M., Daley, M., Gregoire, J.P., Mérette, C., 2006. Epidemiology of insomnia: prevalence, self-help treatments, consultations, and determinants of helpseeking behaviors. Sleep Med. 7, 123-130. http://dx.doi.org/10.1016/ j.sleep.2005.08.008.

Morin, C.M., Rodrigue, S., Ivers, H., 2003. Role of stress, arousal, and coping skills in primary insomnia. Psychosom. Med. 65, 259-267. http://dx.doi.org/10.1097/ 01.psy.0000030391.09558.a3.

Mortier, P., Demyttenaere, K., Auerbach, R.P., Cuijpers, P., Green, J.G., Kiekens, G., Kessler, R.C., Nock, M.K., Zaslavsky, A.M., Bruffaerts, R., 2017. First onset of suicidal thoughts and behaviours in college. J. Affect. Disord. 207, 291-299. http:// dx.doi.org/10.1016/j.jad.2016.09.033.

National Sleep Foundation Sleep and Teens Task Force, 2000. Adolescent sleep needs and patterns: Research report and resource guide. Washington, D.C.

Ohayon, M.M., 2002. Epidemiology of insomnia: what we know and what we still need to learn. Sleep Med. Rev. 6, 97-111. http://dx.doi.org/10.1053/smrv.2002.0186.

Ohayon, M.M., Morselli, P.L., Guilleminault, C., 1997. Prevalence of nightmares and their relationship to psychopathology and daytime functioning in insomnia subjects. Sleep 20, 340-348.

Owens, J., Adolescent Sleep Working Group, Committee on Adolescence, 2014. Insufficient sleep in adolescents and young adults: an update on causes and consequences. Pediatrics 134, e921-e932. http://dx.doi.org/10.1542/peds.20141696.

Pigeon, W.R., Pinquart, M., Conner, K., 2012. Meta-analysis of sleep disturbance and suicidal thoughts and behaviors. J. Clin. Psychiatry 73, e1160-e1167. http:// dx.doi.org/10.4088/JCP.11r07586.

Radloff, L.S., 1977. The CES-D scale: a self-report depression scale for research in the general population. Appl. Psychol. Meas. 1, 385-401. http://dx.doi.org/10.1177/ 014662167700100306.

Ribeiro, J.D., Bender, T.W., Buchman, J.M., Nock, M.K., Rudd, M.D., Bryan, C.J., Lim, I.C., Baker, M.T., Knight, C., Gutierrez, P.M., Joiner, T.E., 2015. An investigation of the interactive effects of the capability for suicide and acute agitation on suicidality in a military sample. Depress. Anxiety 32, 25-31. http://dx.doi.org/10.1002/da.22240.

Roberts, R.E., Duong, H.T., 2013. Depression and insomnia among adolescents: a prospective perspective. J. Affect. Disord. 148, 66-71. http://dx.doi.org/10.1016/ j.jad.2012.11.049.

Roberts, R.E., Roberts, C.R., Chen, I.G., 2001. Functioning of adolescents with symptoms of disturbed sleep. J. Youth Adolesc. 30, 1-18. http://dx.doi.org/10.1023/ A: 1005230820074

Roberts, R.E., Roberts, C.R., Duong, H.T., 2008. Chronic insomnia and its negative consequences for health and functioning of adolescents: a 12-month prospective study. J. Adolesc. Health 42, 294-302. http://dx.doi.org/10.1016/ j.jadohealth.2007.09.016.

Rogers, M.L., Stanley, I.H., Hom, M.A., Chiurliza, B., Podlogar, M.C., Joiner, T.E., 2016. Conceptual and empirical scrutiny of covarying depression out of suicidal ideation. Assessment. http://dx.doi.org/10.1177/1073191116645907.

Rudd, M.D., Rajab, M.H., Orman, D.T., Joiner, T., Stulman, D.A., Dixon, W., 1996. Effectiveness of an outpatient intervention targeting suicidal young adults: preliminary results. J. Consult. Clin. Psychol. 64, 179-190. http://dx.doi.org/ 10.1037/0022-006x.64.1.179.

Russell, D., Peplau, L.A., Cutrona, C.E., 1980. The revised UCLA Loneliness scale: concurrent and discriminant validity evidence. J. Pers. Soc. Psychol. 39, 472-480. http://dx.doi.org/10.1037/0022-3514.39.3.472.

Sateia, M.J., Doghramji, K., Hauri, P.J., Morin, C.M., 2000. Evaluation of chronic insomnia. An American Academy of Sleep Medicine review. Sleep 23, 243-308.

Seelig, A.D., Jacobson, I.G., Smith, B., Hooper, T.I., Boyko, E.J., Gackstetter, G.D., Gehrman, P., Macera, C.A., Smith, T.C., 2010. Sleep patterns before, during, and after deployment to Iraq and Afghanistan. Sleep 33, 1615-1622.

Shekleton, J.A., Rogers, N.L., Rajaratnam, S.M.W., 2010. Searching for the daytime impairments of primary insomnia. Sleep Med. Rev. 14, 47-60. http://dx.doi.org/ 10.1016/j.smrv.2009.06.001.

Shochat, T., Cohen-Zion, M., Tzischinsky, O., 2014. Functional consequences of inadequate sleep in adolescents: a systematic review. Sleep Med. Rev. 18, 75-87. http://dx.doi.org/10.1016/j.smrv.2013.03.005.

Spielberger, C.D., 1989. State-Trait Anxiety Inventory: A Comprehensive Bibliography. Consulting Psychologists Press, Palo Alto, CA.

Spielberger, C.D., 1983. Manual for the State-Trait Anxiety Inventory (STAI). Consulting Psychologists Press, Palo Alto, CA.

Staner, L., 2010. Comorbidity of insomnia and depression. Sleep Med. Rev. 14, 35-46. http://dx.doi.org/10.1016/j.smrv.2009.09.003.

Taylor, J.A., 1953. A personality scale of manifest anxiety. J. Abnorm. Psychol. 48, 285-290.

Van Orden, K.A., Cukrowicz, K.C., Witte, T.K., Joiner, T.E., 2012. Thwarted belongingness and perceived burdensomeness: construct validity and psychometric properties of the Interpersonal Needs Questionnaire. Psychol. Assess. 24, 197-215. http://dx.doi.org/10.1037/a0025358.

Van Orden, K.A., Witte, T.K., Cukrowicz, K.C., Braithwaite, S.R., Selby, E.A., Joiner, T.E., Jr., 2010. The interpersonal theory of suicide. Psychol. Rev. 117, 575-600. http:// dx.doi.org/10.1037/a0018697.

Van Orden, K., Witte, T., Gordon, K., Bender, T., Joiner, T., 2008. Suicidal desire and the capability for suicide: tests of the interpersonal-psychological theory of suicidal behavior among adults. J. Consult. Clin. Psychol. 76, 72-83. http://dx.doi.org/ 10.1037/0022-006X.76.1.72.

Walker, M.P., 2009. The role of sleep in cognition and emotion. Ann. N. Y. Acad. Sci. 1156, 168-197. http://dx.doi.org/10.1111/j.1749-6632.2009.04416.x.

Warner, R.M., 2013. Applied Statistics: From Bivariate Through Multivariate Techniques 2nd ed. SAGE Publications, Thousand Oaks, CA.

Weathers, F., Huska, J., Keane, T., 1991. The PTSD Checklist Military Version (PCL-M). National Center for PTSD, Boston, MA.

Winer, E.S., Nadorff, M.R., Ellis, T.E., Allen, J.G., Herrera, S., Salem, T., 2014a. Anhedonia predicts suicidal ideation in a large psychiatric inpatient sample. Psychiatry Res. 218, 124-128. http://dx.doi.org/10.1016/j.psychres.2014.04.016.

Winer, E.S., Veilleux, J.C., Ginger, E.J., 2014b. Development and validation of the Specific Loss of Interest and Pleasure Scale (SLIPS). J. Affect. Disord. 152-154, 193-201. http://dx.doi.org/10.1016/j.jad.2013.09.010.

Zimmerman, M., Ellison, W., Young, D., Chelminski, I., Dalrymple, K., 2015. How many different ways do patients meet the diagnostic criteria for major depressive disorder? Compr. Psychiatry 56, 29-34. http://dx.doi.org/10.1016/j.comppsych.2014.09.007. 